\title{
The self-serving bias
}

\section{Christoph Bosshard}

Dr. med., Vizepräsident der FMH, Departementsverantwortlicher Daten, Demographie und Qualität / SAQM

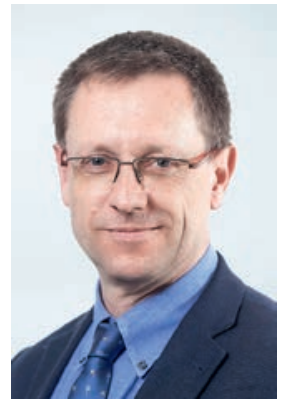

... oder warum wir nie selber schuld sind ... Egal ob Praktikant bei einem internationalen Grossunternehmen, CEO in einem regionalen KMU, als Selbständige oder Angestellter, im privaten oder beruflichem Umfeld, wahrscheinlich ist jeder von uns bereits einmal dem self-serving bias verfallen. Nach einem souveränen Sieg in einem Tennismatch ist die Spielerin überzeugt von ihrem Spiel, den präzisen Schlägen und dass sich das intensive Training vollumfänglich ausbezahlt hat. Verliert der Spieler unerwartet ein Spiel, werden mal weniger, mal mehr äussere Umstände als Argumente präsentiert: der böige Wind liess die Flugbahn des Balls schlecht einschätzen, das Publikum war

\section{Feedback ist eine wichtige Grundlage für die Selbstreflexion und damit für einen kon- tinuierlichen Verbesserungsprozess}

unruhig und störte die eigene Konzentration, dem Schieds- oder Linienrichter unterliefen bei den entscheidenden Punkten Fehler ...

Und genau in solchen und ähnlichen Situationen ist eine akzeptierte und breit verankerte Feedbackkultur zentral. Bill Gates hat dies folgendermassen treffend formuliert: «Deine unzufriedensten Kunden sind deine beste Lernquelle.»

Feedback ist eine wichtige Grundlage für die Selbstreflexion und damit auch für einen kontinuierlichen Verbesserungsprozess - auch im Gesundheitswesen. Es ist Teil von vielen Praxisprojekten, welche sich am
Plan-Do-Check-Act-Zyklus orientieren. Es dient dazu, die umgesetzten Veränderungen $\mathrm{zu}$ analysieren («Check»), um daraus zu lernen. Der Artikel von Prof. Tanja Manser und Dr. med. Johannes Brühwiler in der aktuellen Ausgabe der Schweizerischen Ärztezeitung «Feedback als Qualitätsinstrument» widmet sich genauer dieser Thematik.

Ebenfalls möchte ich Ihnen den Artikel in der Ausgabe vom 18.9.2019 zur Online-Plattform «Guidelines Schweiz - Update 2019» ans Herz legen. Diese Plattform hat nebst der Verbesserung der Transparenz bezüglich anerkannter und verwendeter Guidelines auch zum Ziel, einen interdisziplinären und interprofessionellen Austausch sowie auch einen Diskurs im Rahmen der Anwendung von Guidelines bei Multimorbidität anzustreben. Auch in dieser Thematik ist und bleibt der Plan-Do-Check-Act-Zyklus zentral. Wenn Sie Ihre Guidelines auf der Online-Plattform noch nicht finden: Bis am 31.10.2019 sind Sie eingeladen, die Guidelines auf unserer Plattform zu dokumentieren.

Und zu guter Letzt ein Hinweis in eigener bzw. Ihrer Sache: Am 30.4.2020 prämiert die FMH / SAQM im Rahmen des Innovation Qualité Qualitätsprojekte. Der

\section{Veränderungen analysieren und daraus lernen.}

Themenschwerpunkt fokussiert auf «Feedback als Qualitätsinstrument». Reichen Sie Ihre Qualitätsprojekte bis am 9.12.2019 bei uns ein.

Herzlichen Dank! 